\title{
With thanks to our 2012 peer reviewers
}

We are grateful to the following people for their significant contribution to Chronic Diseases and Injuries in Canada as peer reviewers in 2012. Their expertise ensures the quality of our journal and promotes the sharing of new knowledge among peers in Canada and internationally.

Rebecca Armstrong

Laurent Azoulay

Shelina Babul

Claude Bégin

Claudia Blais

Robert Brison

Mariana Brussoni

Stephanie Burrows

Leslie Campbell

Mary Chipman

Rachel Colley

Sarah Connor Gorber

Michael Cusimano

Fernando De Maio

Jessica Dennis

Helen Edwards

Tewodros Eguale

Mariam El-Zein

Marie-Pierre Gagnon

Alain Gauthier

Julie Green
Philip Groff

Marguerite Guiguet

Ken Johnson

Marie-Jeanne Kergoat

Kyungsu Kim

Malcolm King

Lucie Laflamme

Rachel Lane

Kristian Larsen

Bernard-Simon Leclerc

Gilles Légaré

Bing Li

Lisa Lix

Alice Lytwyn

Alison Macpherson

Ruth Martin-Misener

Ian McDowell

Steven McFaull

Elizabeth McGregor

Larry McKeown

Leia Minaker
Joel Monárrez-Espino

Lisa Oliver

Sai Yi Pan

Louise Parker

Jennifer Payne

William Pickett

Karen Poon

Virginia Powell

Bob Prosser

Brian Rowe

Conor Sheridan

Richard Stanwick

Gerold Stucki

Eva Suarthana

Katherine Teng

Wendy Thompson

Bliss Tracy

Jean-Pierre Villeneuve

Michelle Vine

Kathryn Wilkins

Katrina Zanetti 